\title{
A System for Input of 3D Objects into Computing Environment
}

\author{
Raycho Ilarionov, Krasimir Krastev \\ TU - Gabrovo
}

\begin{abstract}
This paper proposes an approach for design and implementation of automated 3D scanner used for input of mechanical 3D objects into computing environment. The presented model of 3D scanner is based on kinematic diagram of positioning system with 5 degree of freedom -3 linear and 2 rotational, each driven by servo motors. For distance measuring is used laser scanning head with rotational triangulation. The paper describes also algorithms for functional control of the scanning process, obtaining of point cloud, object reconstruction and export to standard CAD format.
\end{abstract}

Keywords - 3D scanning, CAD/CAM, Laser measuring, Positioning System, Point Cloud, 3D object reconstruction.

\section{INTRODUCTION}

Nowadays, the design of new products in mechanical engineering becomes impossible without the use of CAD software for three dimensional modeling. In this relation more topical become the question for direct entry of finished parts into computing environment in order to create theirs models, parameters measurement or just for quality control [9]. This process is known also, as reverse engineering. For its needs are used specialized devices called under common name - 3D scanners. Today this class of devices are a necessary component in each one modern CAD/CAM system in machine building, as they help to improve efficiency in the design and maintenance of new machine elements, as well as such that are out of productions.

Due to its relevance, the systems for 3D scanning are subject of continuous development and improvement [7]. Could be divided into three main directions of development:

- Automation of the scanning process;

- Resolution and scanning speed;

- Detailed scanning;

In this paper is presented an approach for design of system for 3D object scanning with higher level of automation and possibility for many sides scanning of objects with complex geometrical shape with presence of multiple holes, slots and edges.

\section{EXPOSITION}

In the classical scanners, scanning is a process of digitalization of a given object with the purpose of entering a two dimensional graphic information from it into computing environment. Unlike them the 3D scanners captures the surface shells of given object i.e. his relief [4]. The process of entering of three dimensional bodies into computing environment consists of two basic steps - 3D scanning and object reconstruction (Fig. 1.).

The information, which is collected by a 3D scanner, represents a set of points in three-dimensional space, also called 'point cloud'. Each point from the cloud lying on the object surface and is represented by its three-dimensional coordinates - $x, y$ and $z$. In this way can be get a clear picture for relief and shape of the object, and subsequently by using suitable CAD product to be created a model, measurement or analyzing of parameters. As output information from 3D scanning is obtained a file containing the three coordinates of each point from the object surface. The reconstruction of the scanned objects is made by CAD programs which implement specialized algorithms for generation of polygonal mesh and export of the results to standard CAD formats [8].

As input data for the reconstruction of an object is a file generated by $3 \mathrm{D}$ scanner, which contains the set of three-dimensional points describing the scanned object.

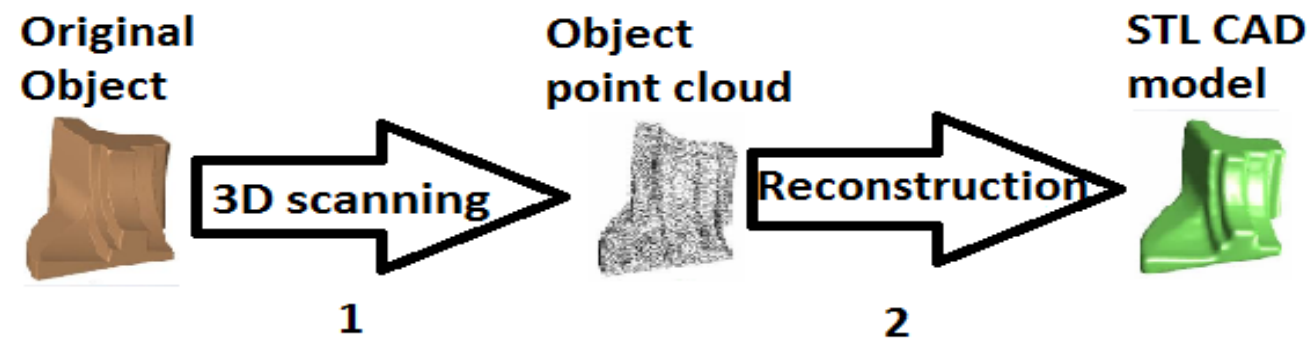

Fig. 1. 3D object input into computing environment 
In general, a 3D scanner consists of a scanning measuring head and positioning electro-mechanical system [4]. The scanning head measures relative coordinates from its current position to the object surface and the positioning system is used to move the head around the object (or vice versa), taking into account the absolute coordinates, compared to defined reference point. By accurate juxtaposing of the relative coordinates from the scanning head with the absolute coordinates from the positioning system, can be obtain a set of $3 \mathrm{D}$ points describing a scanned object [5].

In designing of systems for $3 \mathrm{D}$ scanning it is necessary to be defined two main points:

Maximum dimensions of the scanned objects;

Scanning method and optimal resolution;

The overall dimensions of the scanned objects can vary in very wide range. In the machine building, however most of the components, which are usually subjected to $3 \mathrm{D}$ scanning, fall within the range of up to $300 \mathrm{~mm}$ in height and up to $500 \mathrm{~mm}$ in diameter.

The classical method for 3D scanning uses contact measurement probe to measure the distance to the object surface. This method gives the most accurate results, but is relatively slow and there is a prerequisite of scratching the scanned surface. Modern 3D scanning devices used mainly noncontact scanning methods, as these are more preferred in this type of systems. The principle of their work consists in the emission of light toward the object and calculating the angular offset or the time for receiving of the reflected light. The resolution and accuracy of the scanning heads depends largely on their measuring range and is selected depending on the required accuracy of scanning. In common case this range is up to $100 \mathrm{~mm}$ measurement range, $0.025 \mathrm{~mm}$ resolution and $+/$ $0.005 \mathrm{~mm}$ repeatability.

This paper considers the following key points related to the design of a system for 3D scanning:

Selection of kinematic model for positioning system of the 3D scanner;

Selection of scanning head;

Selection of elements for drive and control of the positioning system;

Structural and functional model of the 3D scanner:

Reconstruction of scanned 3D object and exporting to standard CAD format;

\section{DEVELOPMENT OF KINEMATIC MODEL FOR POSITIONING SYSTEM}

Desktop 2D scanners use two-dimensional coordinate positioning system for perambulating the scanned image. However, for collecting of threedimensional information this is not enough - it is necessary at least one more, third coordinate.

When scanning 3D objects can be considered two main aspects - the movement of the scanning head around and along the object or vice versa - moving the object around and under scanning head [10]. The first option is more appropriate, because of the significantly lower mass and dimensions of the scanning head compared to some parts that may be subjected to scanning.

To unify the very kinematic model of the scanning system it is necessary to approximate every part which should undergo scanning to an object of regular shape. Regular shaped 3D bodies in Cartesian space can be divided into two large groups:

- rotational bodies - they are obtained through rotating a curve around one of the axes;

- $\quad$ plane bodies - they are obtained through linear motion of a curve along one of the axis;

It follows, that are necessary at least four degrees of freedom - three linear for horizontal and vertical motion of the scanning head and one more to rotate the object. In practice, however, the actual threedimensional objects are irregularly shaped with many edges, holes and slots. This entails the necessity of an additional degree of freedom to rotate the scanning head at different angles toward the scanned surface.

A model covering these primitives should possess at least five degree of freedom - three linear and two rotational movements.

The selected kinematical model of positioning system is a five axis coordinate system of portal type for moving scanning head and rotation of the scanned object (Fig. 2.). There are three linear axes ' $\mathrm{X}$ ', ' $\mathrm{Y}$ ', ' $\mathrm{Z}$ ' and two rotational axes - ' $\mathrm{A}$ ' and ' $\mathrm{B}$ '.

Table 1 shows the nominal parameters of the presented kinematic model for positioning system of the 3D scanner.

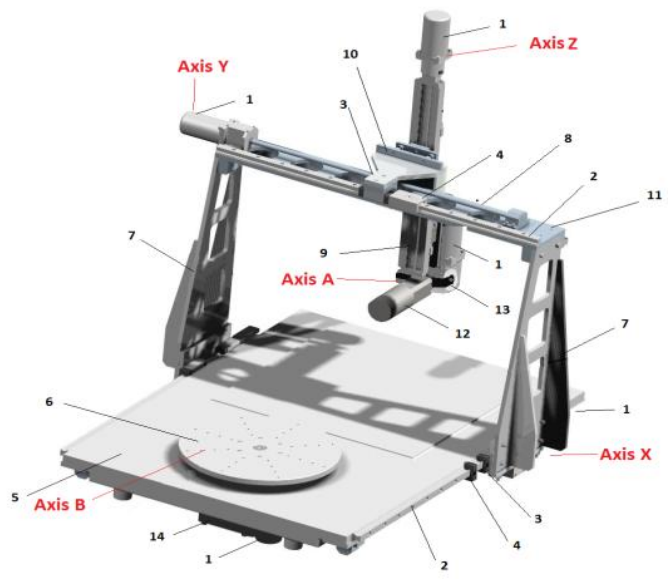

Fig.2. Kinematic model of the positioning system

Elements of the positioning system:

Driving servo motors for ' $\mathrm{X}$ ', ' $\mathrm{Y}$ ', ' $\mathrm{Z}$ ', ' $\mathrm{A}$ ' and 'B' axes;

2. Guiding rails;

3. Sliding carriage;

4. Linear encoder;

5. Basis;

6. Rotational table (axis ' $\mathrm{B}$ ');

7. Vertical support;

8. Ball-screw - axis ' $\mathrm{Y}$ '; 
9. Ball-screw - axis 'Z';

10. Carrier - axis ' $Z$ ';

11. Horizontal support;
12. Scanning head;

13. Reducer - axis ' $A$ ';

14. Reducer - axis 'B';

TABLE. 1.

NOMINAL PARAMETERS OF THE POSITIONING SYSTEM

\begin{tabular}{|l|l|l|l|l|l|}
\hline Axis & Working range & Work Speed & Max. Speed & Nom. Torque & Min. Step \\
\hline$X$ & $550 \mathrm{~mm}$ & $5000 \mathrm{~mm} / \mathrm{min}$ & $10000 \mathrm{~mm} / \mathrm{min}$ & $0,25 \mathrm{Nm}$ & $0,001 \mathrm{~mm}$ \\
\hline$Y$ & $550 \mathrm{~mm}$ & $5000 \mathrm{~mm} / \mathrm{min}$ & $10000 \mathrm{~mm} / \mathrm{min}$ & $0,18 \mathrm{Nm}$ & $0,001 \mathrm{~mm}$ \\
\hline$Z$ & $350 \mathrm{~mm}$ & $5000 \mathrm{~mm} / \mathrm{min}$ & $10000 \mathrm{~mm} / \mathrm{min}$ & $0,19 \mathrm{Nm}$ & $0,001 \mathrm{~mm}$ \\
\hline A & $+/-90^{\circ}$ & $1800 \% \mathrm{~min}$ & $3600 \% \mathrm{~min}$ & $0,23 \mathrm{Nm}$ & $0,01^{\circ}$ \\
\hline$B$ & $0-360^{\circ}$ & $3600 \% \mathrm{~min}$ & $7200 \% / \mathrm{min}$ & $0,32 \mathrm{Nm}$ & $0,01^{\circ}$ \\
\hline
\end{tabular}

*The presented torque of axis ' $\mathrm{X}$ ' is for one of the driving axis at full load.

The position accuracy is achieved by incremental linear encoders with resolution of 1000inc per mm, which allows position accuracy of $1 \mu \mathrm{m}$. The kinematic scheme is comparatively light without any major inertial moments. The portal type provides stability of the system to the vibrations of the measuring $\mathrm{Z}$ coordinate. Synchronous drive of the portal prevents twisting and guarantees the perpendicularity of axis ' $\mathrm{Y}$ ' to axis ' $\mathrm{X}$ '. For driving of the linear axes are used precise ball-screws with $2 \mathrm{~mm}$ pitch and driving of the rotary axes is through reducers. Reduction factor for axis ' $A$ ' is $i=10$, and for axis ' $\mathrm{B}$ ' is $\mathrm{i}=100$. Presented in the table nominal torques are calculated from the nominal weight of the elements in corresponding axis, maximal positioning speed and permissible acceleration - up to $20000 \mathrm{~mm} / \mathrm{s} 2$ for linear axes and $3000 \% / \mathrm{s} 2$ for rotational axes. Maximum allowed mass of the scanned objects placed on the rotary table is $20 \mathrm{~kg}$.

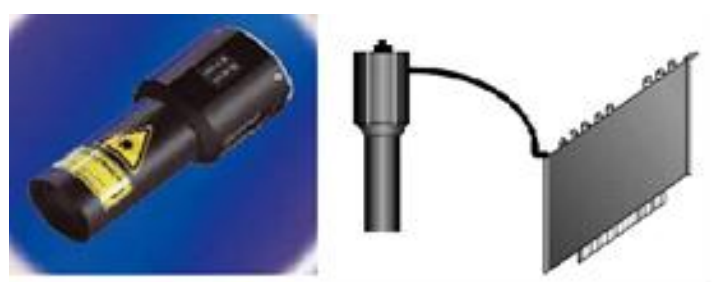

Fig. 3. Laser scanning head OTM $3 \mathrm{~A}$

\section{SELECTION OF SCANNING HEAD}

The selection of scanning head usually is individual depending of the specific group of three-dimensional objects, which has to be scanned.

For this selection are necessary to be observed the following main criterions:

Scanning head working principle;

Measurement range ;

Resolution;

Repeatability of measurement;

Time to measure;
Type of the communication interface;

At the moment most spread measurement methods [6] used in scanning heads are:

Structured light - projecting of light patterns over the object surface with using of DLP projector and image capturing by CCD camera;

Laser triangulation - projecting of laser light formed as spot or line over the object surface with using of laser source and capturing of the reflected light by CCD sensor;

Both methods use triangulation principle to measure the distance to the object surface (emitter, object and receiver form a triangl) Because of their small masses, scanning heads which operate on the laser triangulation principle are most appropriate for $3 \mathrm{D}$ scanning systems, with motion of the scanning head. Highest scanning speed can be achieved by the laser section method - the projected laser light is line shaped, as by this way are simultaneously scanned up to 10000 points from the object surface. The disadvantage, however, is the strongly expressed shadow effect due to the large angle of triangulation. Rotational triangulation is a kind of laser triangulation, which works at very small base angle between emitter and receiver, as in this way shadowing effect is minimal.

Due to the necessity for scanning of complex geometrical objects with presence of multiple sharp edges and deep slots is selected laser scanning head of Wolf\&Beck GmbH type OTM 3A-50 (Fig. 3) operating on the rotational triangulation method [3]. The scanning head is controlled by PCI controller, which is mounted in PC slot, communication between them is through digital serial interface - RS422 (Fig. 3.). PCI controller is supplied with drivers for Windows. Key features of OTM 3A-50:

- Work distance: $140 \mathrm{~mm}$;

- Measurement range: $50 \mathrm{~mm}$;

- Resolution: $0.005 \mathrm{~mm}$;

- Repeatability: $0.025 \mathrm{~mm}$;

- $\quad$ Time to measure: $500 \mu \mathrm{s}$

Max. slope of the scanned surface: $60^{\circ}$; 
PCI controller of the scanning head provides digital I/O interface for control and synchronization with external systems. Due to its direct action this interface is suitable for synchronization the measurements with the positioning system of the scanner.

\section{SELECTION OF DRIVE AND CONTROL ELEMENTS}

For driving of the positioning system are necessary executive electrical motors, these are stepper motors, DC servo motors and AC servo motors. AC servo motors are preferred choice due to their wide control capabilities, high dynamic, overload capacity and lack of maintenance (in fact, they are eternal).

The selection of driving motors is made on two main criterions:

Necessary torque to drive the positioning system;

Maximal positioning speed;

To unify the model of positioning system it is preferred a selection of same kind servo motors for all axes. Table 1 show, that highest drive torque is needed for axis ' $\mathrm{B}$ ' $-0,32 \mathrm{Nm}$. When choosing the right servo motor it is necessary to provide $25 \%$ of stock. The nominal speed which must provide selected servo motor type is calculated from the maximal positioning speed and pitch of the screw for linear axes and reduction factor for the rotational axes. In this case is derived that, for driving of all linear axes is required nominal speed $>5000 \mathrm{~min}-1$, for axis ' $\mathrm{A}$ ' >100 min-1 and for axis ' $\mathrm{B}$ ' $>2000 \mathrm{~min}-1$.

For the concrete application are selected synchronous servo motors type DT3 of the German company AMK GmbH [2] (may be used and the servo motors of different manufacturer). In Table 2 are presented technical parameters of this kind of servomotors.

The total number of servo motors is six. Two of them are used to drive the portal part along ' $\mathrm{X}$ ' axis and by one to drive of axes ' $Y$ ', ' $Z$ ',' $A$ ' and 'B'.

For control of the selected servo motors are used double servo inverter type KWD with nominal power of $2 \mathrm{x} 1 \mathrm{kV}$. This selection is made based on servo motors nominal current, as is provided $25 \%$ stock. The power supply of the servo inverters is by common module type KEN10 with nominal power of $10 \mathrm{kVA}$. Control plates for each axis are KW-R06 type with integrated EtherCAT control interface, working in real time.

For positioning system control is selected PC based industrial controller (PLC) of AMK GmbH Company - type A5S-ECO with EtherCAT digital interface.

TABLE. 2.

TECHNICAL PARAMETERS OF SELECTED DT3 SERVO MOTOR BY AMK GMBH

\begin{tabular}{|l|l|l|l|l|l|l|l|l|}
\hline Motor type & $\begin{array}{l}\mathrm{MO} \\
{[\mathrm{Nm}]}\end{array}$ & $\begin{array}{l}\mathrm{MN} \\
{[\mathrm{Nm}]}\end{array}$ & $\begin{array}{l}\mathrm{PN} \\
{[\mathrm{kW}]}\end{array}$ & $\mathrm{I}_{\mathrm{N}}(\mathrm{A})$ & $\mathrm{n}_{\mathrm{N}}[\mathrm{U} / \mathrm{min}]$ & $\begin{array}{l}\mathrm{n}_{\max } \\
{[1 / \mathrm{min}]}\end{array}$ & $\mathrm{M}_{\max }[\mathrm{Nm}]$ & $\mathrm{Imax}[\mathrm{A}]$ \\
\hline DT3-0,5-10-RxO-9000 & 0,64 & 0,5 & 0,31 & 0,9 & 6000 & 10000 & 1,9 & 4 \\
\hline
\end{tabular}

\section{STRUCTURAL AND FUNCTIONAL MODEL OF THE 3D SCANNER}

\section{Structural model}

The structural model of the 3D scanner (Fig. 4.) represents all connections between the elements in the control system. It consists of two control subsystems:

PC-CAD work station with Windows based operation system. Include:

Program for 3D scanner control, as Windows application;

PCI controller for the scanning head PCI OTM3A mounted in slot of PC;

Driver for scanning head control;

CAD software for edit and reconstruction of the scanned objects;

System for driving and control of the positioning system. Include:

Industrial controller for real time motion control;

Application program, which implements motion control functionality;

6 servo motors for driving of axes ' $\mathrm{X}$ ', ' $\mathrm{Y}$ ', ' $\mathrm{Z}$ ', 'A' и 'В';

3 double servo inverter modules for controlling each of the 6 servo motors;
Linear encoders for position measurement by axes ' $\mathrm{X}$ ', ' $\mathrm{Y}$ ', ' $\mathrm{Z}$ ' and rotational encoder for position measurement by axis ' $\mathrm{B}$ '.

All motion control functions of the positioning system are executed by Motion Controller AMK A5$\mathrm{ECO}$, as is guaranteed real time operation with higher accuracy of synchronization between all servo axes at fixed transmitting cycle of $500 \mu \mathrm{s}$ and minimal dissipation of 500ns. The connection between motion control and the servo drives is through digital EtherCAT interface. The connection between the two subsystems is through Ethernet (LAN) interface.

Synchronization between scanning head measurements and actual coordinates from the positioning system is through a clock signal, generated at each measurement from a digital output of the OTM3A PCI controller [5]. This clock signal is send to trigger input for position measurement of each servo drive, which cause immediate record of the current axis position with corresponding sequential number in the PLC memory. In the same time measured data by the scanning head are recorded in the memory of the work station (PC) with corresponding sequential number. Buffer length is up to 125000 records organized as FIFO structure, at overflow it starts from the beginning. Buffer structure 
and data format, which are recorded by the scanning head for each captured point are presented in Table 3 and the data format from the positioning system in Table 4.

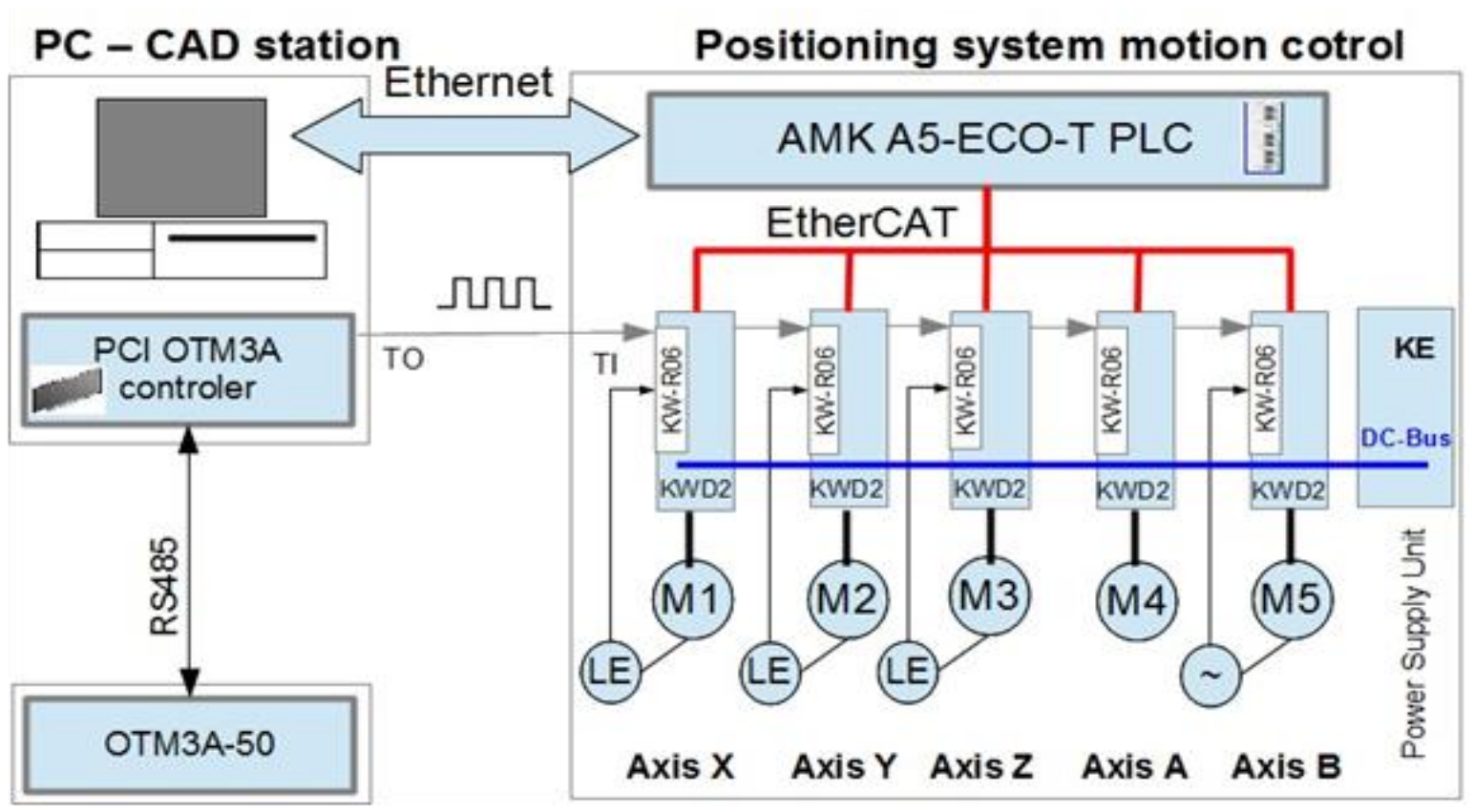

Fig. 4. Structural model of the 3D scanner

TABLE. 3.

SCANNING HEAD - STRUCTURE OF THE DATA BUFFER

\begin{tabular}{|l|l|l|l|l|}
\hline Distance[0] & Angle[0] & Intensity[0] & Status[0] & Tan[0] \\
\hline Distance[1] & Angle[1] & Intensity[1] & Status[1] & Tan[1] \\
\hline & & & & \\
\hline Distance[n] & Angle[n] & Intensity[n] & Status[n] & Tan[n] \\
\hline
\end{tabular}

TABLE. 4.

POSITIONING SYSTEM - STRUCTURE OF THE DATA BUFFER

\begin{tabular}{|l|l|l|l|l|}
\hline $\mathrm{X}[0]$ & $\mathrm{Y}[0]$ & $\mathrm{Z}[0]$ & $\mathrm{A}[0]$ & $\mathrm{B}[0]$ \\
\hline $\mathrm{X}[1]$ & $\mathrm{Y}[1]$ & $\mathrm{Z}[1]$ & $\mathrm{A}[1]$ & $\mathrm{B}[1]$ \\
\hline & & & & \\
\hline $\mathrm{X}[\mathrm{n}]$ & $\mathrm{Y}[\mathrm{n}]$ & $\mathrm{Z}[\mathrm{n}]$ & $\mathrm{A}[\mathrm{n}]$ & $\mathrm{B}[\mathrm{n}]$ \\
\hline
\end{tabular}

A minimal scanning step of $0,041 \mathrm{~mm}$ is calculated at maximal scanning speed of OTM 3A-50 up to 2000p/s and maximal moving speed of the positioning system up to $5000 \mathrm{~mm} / \mathrm{min}$.

\section{Functional model}

The functional model of the 3D scanner (Fig. 3.) considers the functionality and the tasks of the control software. The software is divided into two main control programs, which must work together:

Control program of the 3D scanner. Developed with QT Creator and MinGW compiler. Provides the following functionality:

Interactive graphical interface to the operator for working with the 3D scanner;

Control of the laser scanning head;

Control of the positioning system with sending of commands to the PLC;

Maintain communication with the PLC;

Control of the 3D scanner operating modes;
Collecting and recording of data from the scanning; Application program for motion control. Developed with CoDeSys V2.3 [1]. Provides the following functionality:

Control and diagnostic of all servo motors;

Referencing of axes ' $\mathrm{X}$ ', ' $\mathrm{Y}$ ', ' $\mathrm{Z}$ ', 'A' and 'B';

Manual positioning of all axes - continuous and in step mode;

Input of settings for scanning;

Automatic scanning by surface primitive presented by the control program;

Record of measured coordinates in memory buffer and their transfer to a $\mathrm{PC}$;

Automatic monitoring and maintenance in the measuring range of the scanning head;

The graphical user interface (Fig. 5.) of the program for control the scanner, provides opportunity to work in two operating modes: 


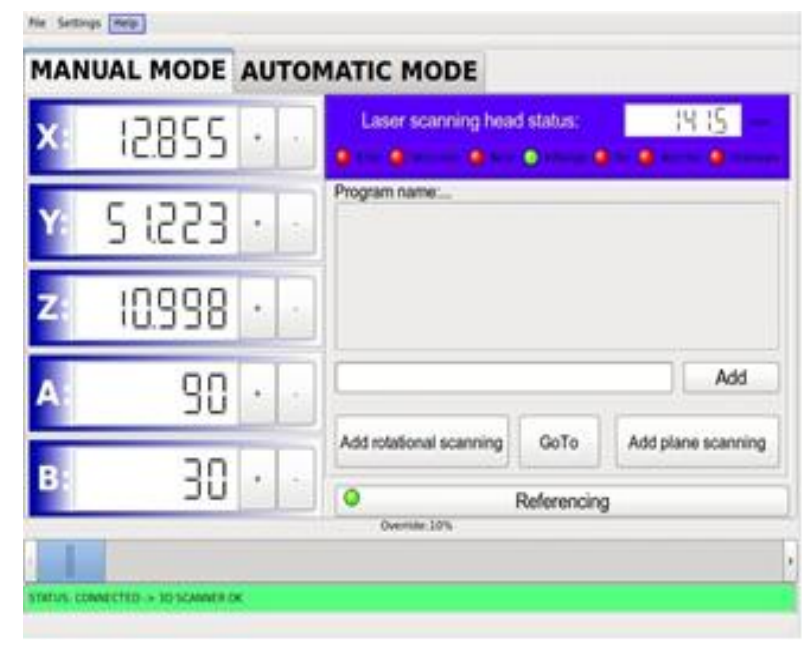

Fig. 5. Graphical user interface of the 3D scanner control program

Manual mode - in this operating mode the user interface provides:

Manual positioning with buttons of each selected axes in continuous motion control or in stepping motion control and sending of commands for axes referencing;

Indication of current position and measured distance by the scanning head;

Menu for system settings - for scanning head and the positioning system;

Command interpreter and program editor;

Wizard with graphical user interface for entering of scanning cycles;

Status information of the positioning system and laser scanning head;

Automatic mode - this operating mode is responsible for the automatic scanning by preselected input program. In automatic mode the user interface provides:

Start of preselected scanning program;

Buttons for start, stop and pause of started automatic cycle;

\section{Format of the commands:}

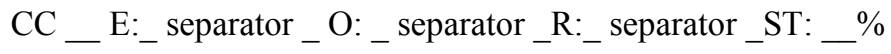

TABLE. 5.

SET OF PROGRAM CYCLES FOR SCANNING OF SURFACE PRIMITIVES FROM 3D OBJECT

\begin{tabular}{|l|l|}
\hline Command & Meaning \\
\hline TA & Absolute positioning, target coordinates are set with E: \\
\hline TR & Relative positioning, target coordinates are set with E: \\
\hline PR & Plain rectangle area scanning, O: set the origin point, E: end point - by diagonal \\
\hline PC & Plain circle scanning, O: set the point of circle center, E: end point - from lying on the circle \\
\hline PA & Plain scanning as part of circle O: set the arc origin point, E: end point, R-radius of the arc \\
\hline PT & Plain triangle area scanning (isosceles), O: set first corner - origin point, E: set second corner \\
\hline CL & Cilindrical scanning, O: set the origin point, E: set the end point \\
\hline CS & Cilindrical scanning with offset center * - O: set origin point, E: set end point, R: set the offset \\
\hline SF & Spherical scanning, O: set the origin point, E: set end point, R set the radius \\
\hline
\end{tabular}




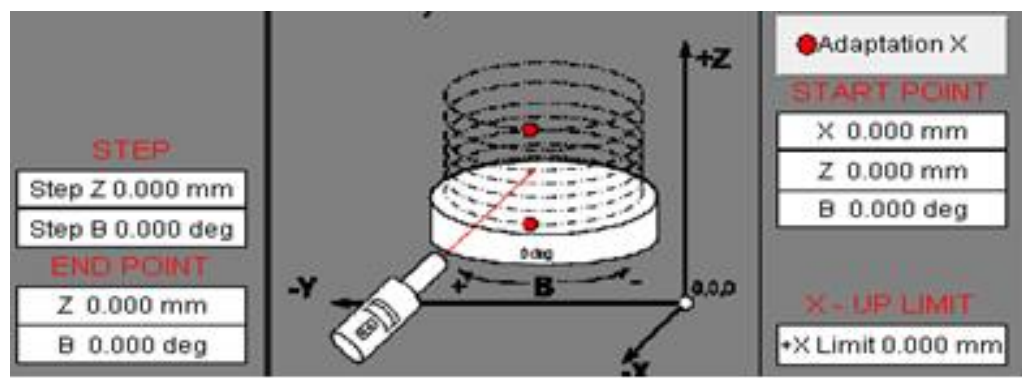

Fig. 6. Window of the wizard for input of cylindrical scanning cycle

Each command for scanning cycle begins with twoletter mnemonic code - ' $\mathrm{CC}$ '. The command is followed by parameters for the scanning - end coordinates (End) 'E:', start coordinates (Origin) 'O:', optional parameter 'R:', scanning step 'ST:' and speed override ' $\%$ ', as percent of the nominal.

For example: PR E: X100.0 Y20.0 A90.0 \# O:X0.0 Y0.0 A90.0 \# ST:0.01\%100 - plane scanning in XY plane from $0.0,0.0$ to $100.0,20.0$ with step of $0.01 \mathrm{~mm}$, at full speed. Required for each parameter group is to be separated with sign for separation - '\#'. The format of each parameter is up to three coordinates. The scanning plane is selected depending of the first two coordinates, stored after ' $\mathrm{E}:$ ' - end point parameter. The first coordinates stored after 'E:' define the master axis for the cycle and the second coordinate is an auxiliary - slave axis is controlled in stepping mode depending of the scan step. Depending of the difference between first and last coordinates, third axis is interpolated together with the master axis - if exists a record. The origin coordinates are not mandatory, if they are missing, as point of origin is accepted the current position of the scanner. Setting of scanning step is required only at the first command in the program - it is modal. If no speed is set, the system runs at maximum speed.
During the scanning process the two data flows are combined together in a common file by the control program - the data are shown in Table 3 and Table 4. This file is stored in the same folder on PC, where is placed the control program. After end of the scanning a coordinate transformation is applied over the recorded data, with purpose to convert the measured distance data and the captured positions of the positioning system in three-dimensional Cartesian coordinates.

Full coordinate transformation of the 3D scanner is presented with dependence (1) and it is universal for all methods for scanning of three dimensional objects with the presented prototype.

Where:

$\theta_{i}-$ is the angle of rotation of the scanning head(axis 'A');

$\alpha_{i}$ - is the angle of the rotational table (axis 'B');

$r_{i}$ - is the difference between ' $\mathrm{X}$ ' axis position and the measured distance by the scanning head in sequential point of measurement, taking into account ' $A$ ' axis position;

$j_{i}-$ is the distance from the axis of symmetry of axis ' $\mathrm{B}$ ' to the object surface in the point of measurement;

$$
P=\left[\begin{array}{ccc}
\mathrm{x}_{1} & \mathrm{y}_{1} & \mathrm{z}_{1} \\
\mathrm{x}_{2} & \mathrm{y}_{2} & \mathrm{z}_{2} \\
\cdots & \cdots & \cdots \\
\mathrm{x}_{\mathrm{n}} & \mathrm{y}_{\mathrm{n}} & \mathrm{z}_{\mathrm{n}}
\end{array}\right]=\left[\begin{array}{cccc}
r_{1} \cos \left(\alpha_{1}+\arctan \frac{j_{1}}{Y_{1}}\right) & r_{1} \sin \left(\alpha_{1}+\arctan \frac{j_{1}}{Y_{1}}\right) & Z_{1}-\left(l_{1} \sin \theta_{1}\right) \\
r_{2} \cos \left(\alpha_{2}+\arctan \frac{j_{2}}{Y_{2}}\right) & r_{2} \sin \left(\alpha_{2}+\arctan \frac{j_{2}}{Y_{2}}\right) & Z_{2}-\left(l_{2} \sin \theta_{2}\right) \\
r_{n} \cos \left(\alpha_{n}+\arctan \frac{j_{n}}{Y_{n}}\right) & r_{n} \sin \left(\alpha_{n}+\arctan \frac{j_{n}}{Y_{n}}\right) & Z_{n}-\left(l_{n} \sin \theta_{n}\right)
\end{array}\right]
$$

As output data from the scanning is obtained standard file in *.xyz or *.pcd format, which contains the point cloud and fully describe the scanned 3D object.

\section{RECONSTRUCTION OF SCANNED 3D OBJECTS}

For reconstruction of the scanned objects with proposed prototype of 3D scanner has been chosen the program 'MeshLab' - developed by Computing Lab IST CNR [8]. MeshLab supports multiple of functions for editing and processing of point clouds with $3 \mathrm{D}$ points as end large scale of algorithms for generating of polygonal meshes, rendering and finally export of the models into STL or DXF CAD file formats. 

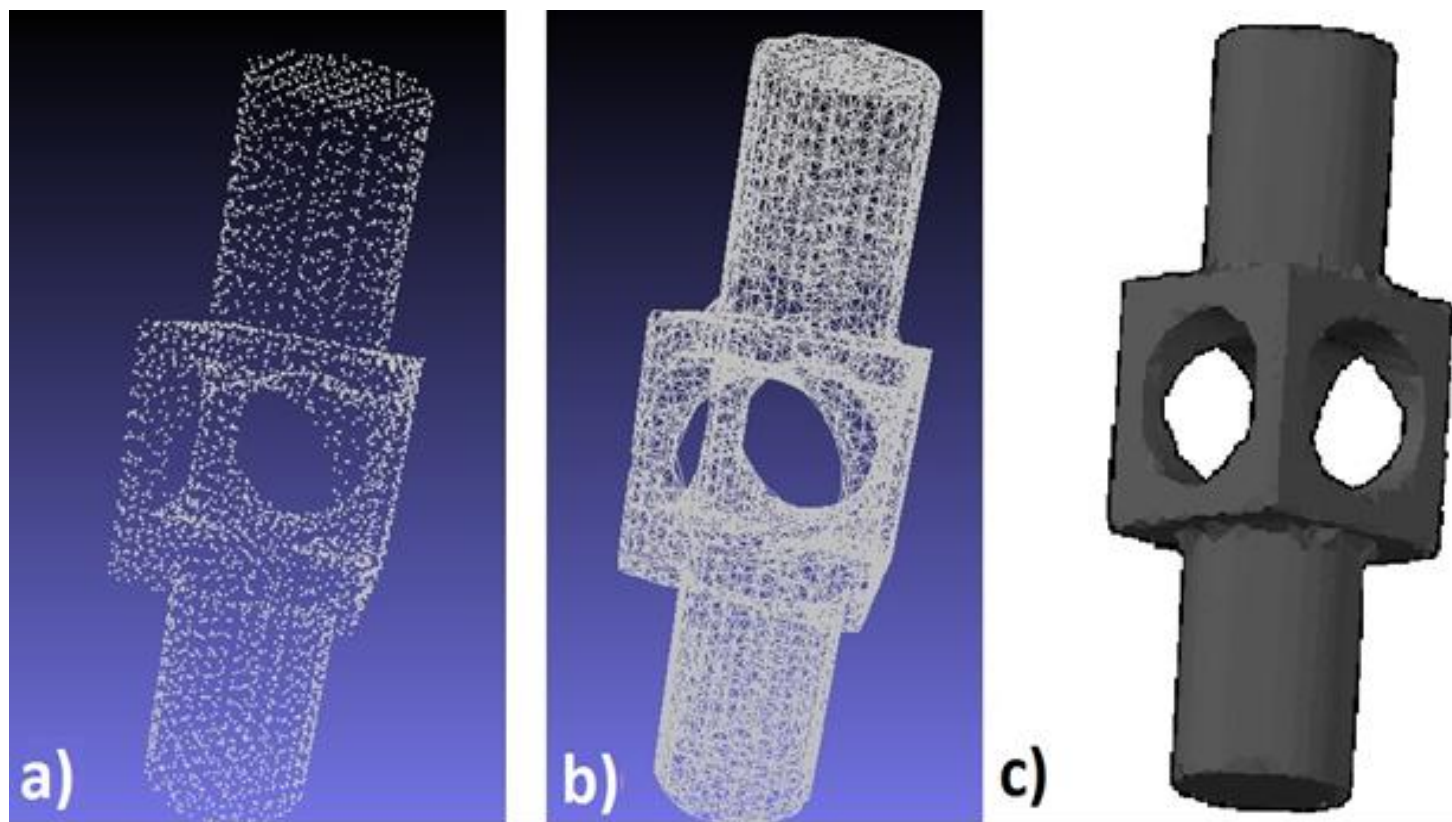

Fig. 7. a) Point cloud of scanned mechanical detail, b) Triangulated model, c) Solid model in AutoCAD

The process of reconstruction passes through the following steps:

Import and editing of the point cloud - includes basic operations, as shifting, rotation, grouping, scaling, as well as noise reduction and the excess point in the cloud.

Construction of polygons - includes polygonal meshes generation, editing, optimization and smoothing. Some of the most used algorithms are:

Alpha Complex/Shape;

Delaunay Triangulation;

Surface Reconstruction-Ball-Pivoting;

Surface Reconstruction - Poisson;

Voronoi Filtering;

Creation of NURBS surfaces - includes processing and creation of NURBS surfaces, surfaces grouping, editing and finishing.

Exportation of the models into standard CAD file format - most used are STL and DXF file formats.

On Fig.7 consistently are shown the stages of reconstruction of scanned object with the proposed model of 3D scanner.

As reconstruction input data has imported a *.xyz file into MeshLab, which contains the point cloud of the scanned object. The scanning has made with resolution of $0.075 \mathrm{~mm}$. For creating of polygonal mesh has used the algorithm "Voronoi Filtering" with maximum poles threshold of 100 .

Good results are obtained also with algorithms "Alfa Complex/Shape" and "BallPivoting". For smoothing of the created surfaces and detailing of the object has applied algorithm with the method of "Catmull-Clark". Finally the obtained polygonal model has exported into AutoCAD, where is presented as a solid body.

\section{CONCLUSION}

The proposed in this paper approach for designing of 3D laser scanning device has been realized, as prototype in AMK LLT Company, Bulgaria, together with the support of Technical University of Gabrovo.

In the design process have been consistently considered the criteria for selection of suitable kinematic scheme, choice of scanning head, drive and control elements.

The presented prototype of 3D scanner is intended mainly for scanning of three-dimensional solid bodies in machine building, which have complicated form with multiple edges and slots. This has been obtained by using a 5-axis positioning system that provides linear motion and rotation of the measuring element as well vertical rotation of the scanned object. The selected laser scanning head works on the principle of rotational triangulation and allows scanning of surfaces at slope up to $60^{\circ}$ and presence of transparent and highly reflective areas.

Proposed is a method for separation of the object surface shell into primitives for scanning as separate from it areas, which are then combined.

The maximal dimensions of the scanned objects are up to $300 \mathrm{~mm}$ in height and $500 \mathrm{~mm}$ in diameter, the maximum weight of the parts is $20 \mathrm{~kg}$. A satisfactory accuracy of $0.025 \mathrm{~mm}$ of the scanning and minimal positioning step of $1 \mu \mathrm{m}$ for the linear axes and $0.01^{\circ}$ for the rotational axis, has been achieved.

As output data from the scanning have been obtained standard files in *.xyz or *.pcd formats containing the set of the scanned points lying on the object surface. 
Ilarionov R., Krastev K. A SYSTEM FOR INPUT OF 3D OBJECTS INTO COMPUTING ENVIRONMENT

Also has been proposed methodology for reconstruction of $3 \mathrm{D}$ objects by theirs point clouds and export of them as standard file formats into CAD environment, with using of the program MeshLab.

\section{REFERENCES}

[1] [1] 3S-Smart Software Solutions GmbH. User Manual for PLC Programming with CoDeSys 2.3 . 2007.

[2] [2] AMK GmbH. Servo drives user manuals. 2005.

[3] [3] Dr. Wolf\&Beck GmbH. Integration Handbook for Optoelectronic Distance Measuring System, 2005.

[4] [4] Ilarionov, R., Model approach in the design of device for 3D information input into computing environment, CompSyst Tech 09, ACM, New York, USA
[5] [5] Ilarionov.,R., K. Krastev, Flexible control of positioning system and laser scanning probe with data flow synchronization, Unitech 12, Gabrovo, Bulgaria

[6] [6] Shell, R., Hall, E. (2000). Handbook of Industrial Automation, Marcel Dekker Inc, New York, USA.

[7] [7] Schäfter + Kirchhoff. Applications: Measuring and Process Control in 3D using Laser Lines, Laser Spots and Laser Patterns, 2011

[8] [8] MeshLab - online documentation. http://sourceforge.net/apps/mediawiki/meshlab/index.php?title =Main_Page

[9] [9] Benefits of 3D http://www.applications3d.com/Blog/?paged=3

[10] Six axis motion control http://www.freepatentsonline.com/8249823.pdf scanning. aparatus. 